\title{
Dataset Paper \\ Discovery of MicroRNAs in Cardamom (Elettaria cardamomum Maton) under Drought Stress
}

\author{
N. Anjali, F. Nadiya, Jinu Thomas, and K. K. Sabu \\ Jawaharlal Nehru Tropical Botanic Garden and Research Institute (JNTBGRI), Palode, Thiruvananthapuram 695562, India
}

Correspondence should be addressed to K. K. Sabu; sabu@jntbgri.res.in

Received 31 December 2016; Revised 21 March 2017; Accepted 19 April 2017; Published 31 July 2017

Academic Editor: Michael Hackenberg

Copyright (C) 2017 N. Anjali et al. This is an open access article distributed under the Creative Commons Attribution License, which permits unrestricted use, distribution, and reproduction in any medium, provided the original work is properly cited.

\begin{abstract}
MicroRNAs (miRNAs) are endogenous, 19-24-nucleotide-long, noncoding RNAs found in animals, plants, and some viruses. Drought is one of the major limiting factors that negatively affect productivity of plants. To date, nothing is known about the regulatory roles of miRNAs in response to drought stress in cardamom. Cardamom collected from the natural forest area in Therakkudi in the Idamalayar forest range was selected for the study. Total RNA was isolated from leaves and stems of control and drought treated cardamom plant, which was used for library construction. Ion torrent sequencing of the two small RNA libraries prepared from plants raised under well irrigated and drought stressed treatments, respectively, created 3,938,342 and 4,083,181 primary reads. A total of 150 conserved and 20 novel microRNAs were identified from both the control and the treated libraries. Discovery of 17 differentially expressed miRNAs under drought stress suggests that these might have been involved in various biological processes to improve plant tolerance to water stress. This study is the first report of drought responsive miRNAs and their targets in cardamom. The outcome of this research could provide insights into the miRNA based regulatory mechanisms in response to drought stress in monocot plants.
\end{abstract}

\section{Introduction}

MicroRNAs (miRNAs), which are functionally significant, noncoding RNAs (approximately 19-24 nucleotides (nt) long), act as posttranscriptional gene expression regulators by being involved in different processes like apoptosis, stress response, and differentiation and different disease conditions [1]. Sequencing is the base for awareness about the number, diversity, expression, and probable roles of small noncoding RNAs in plants [2]. Sanger sequencing or chain termination method has been the dominant method of DNA sequencing which was developed by Frederick Sanger in 1977. Genomic research has become revolutionized with the launch of nextgeneration sequencing (NGS) technologies since 2005. NGS is more economical than Sanger sequencing, and hence researchers are now able to carry out many experiments which were formerly problematic. Cardamom cultivation has good production potential but the plants are vulnerable to many pests, diseases, and abiotic stress factors like droughts, floods, extreme temperatures, salinity, nutrition starvation, and oxidative and heavy metal stress. Drought is one of the major limiting factors that negatively affect the crop productivity of the plant. To resist this drought stress, plants execute several mechanisms at the molecular and physiological levels $[3,4]$. Knowledge on the mechanism behind plant response to drought will be useful for better productivity [5].

\section{Methodology}

Wild cardamom (accession number: TBG-C75) collected from the natural forest area in Therakkudi in the Idamalayar forest range ( $\mathrm{N} 10^{\circ} 13^{\prime} 13.20^{\prime \prime}$ and $\left.\mathrm{E} 76^{\circ} 47^{\prime} 03.7^{\prime \prime}\right)$ in Kerala state of India was selected for the study. The collected plants are maintained in a greenhouse with daily watering (voucher specimen deposited in JNTBGRI Herbarium as TBGT86201). Out of those plants, one group was labeled as "control" and another as "treated." Water was withheld to the plants labeled as "treated" in order to begin the experiment. Stress indications like leaf rolling appeared three weeks after water withholding and at this drought condition the absolute moisture content of the soil was calculated to be $<4.5 \%$. Normal watering was given only to the control plant. 


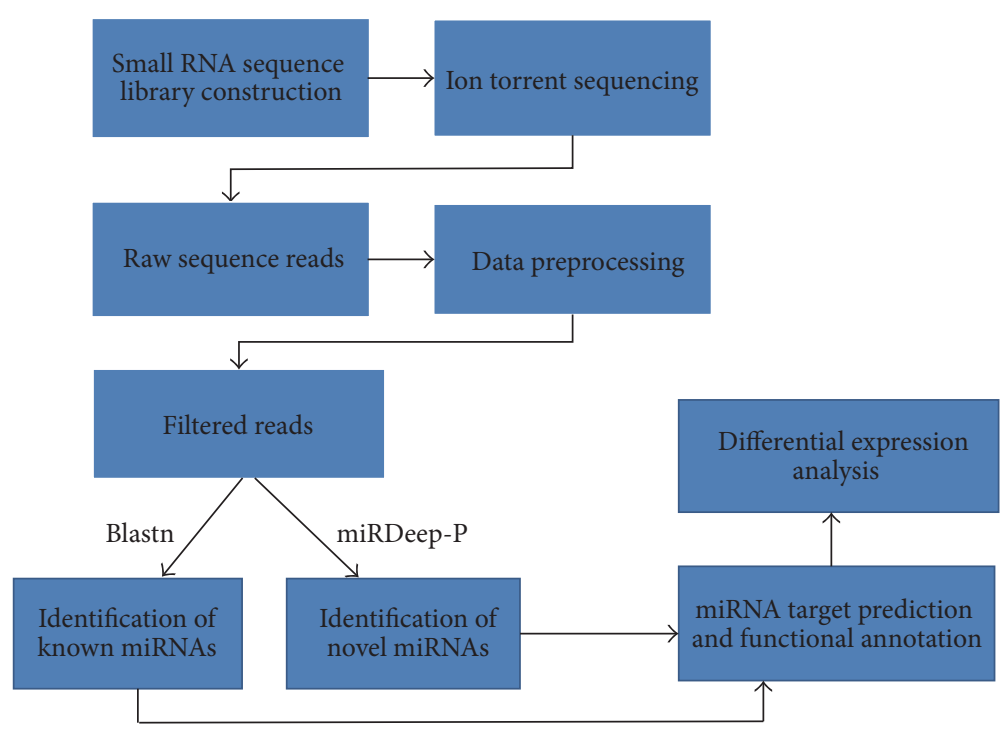

FIGURE 1: The pipeline used for predicting miRNAs from raw small RNA reads of cardamom using various bioinformatics tools.

Small RNA library preparation and next-generation sequencing were as follows. Total RNA was isolated from leaves and stems of control and drought treated cardamom using the combined miRNeasy Mini Kit and CTAB method [6]. Ion total RNA Seq. Kit V2 was used for small RNA sequence library construction following the manufacturer's instructions after pooling the total RNA from leaf and stem tissues in both control and treated samples. The purified libraries were used for sequencing analysis with the ion torrent sequencer by the Centre for Cellular and Molecular Platforms (C-CAMP, Bangalore).

miRNA identification and target prediction were as follows. miRNAs were predicted from the raw small RNA reads using various bioinformatics tools and the pipeline used is depicted in Figure 1. Adaptors were removed from the raw sequencing reads using Cutadapt with error rate (-e) set to 0.1 [7]. The remaining reads were checked against snRNAs, snoRNAs, rRNAs, and tRNAs from NCBI database and the perfect matches were eliminated using Bowtie alignment tool [8]. Reads with 17-27 nt length were kept for further analysis. The identical sequences were collapsed into a single sequence by FASTQ/A Collapser tool available in the FASTX-Toolkit. A Blastn search was performed against plant mature miRNAs from miRBase database to identify conserved miRNAs in cardamom small RNA libraries. The remaining reads were mapped onto the transcriptome sequence of Curcuma longa, the closest relative of cardamom, for the identification of novel miRNAs, as the whole genome or transcriptome sequence of cardamom is not available. The aligned reads were used as input to predict novel miRNAs with the software miRDeep-P (Dataset Item 1 (Table)) [9]. Targets of all the cardamom miRNAs determined in this study were predicted using the psRNATarget (http://bioinfo.noble.org/psRNATarget/) software with default parameters (Dataset Item 2 (Table)) [10]. The model plant Arabidopsis thaliana was selected from the input list of cDNA library in the psRNATarget tool as the reference genome. Singular Enrichment Analysis (SEA) tool from agriGO toolkit (http://bioinfo.cau.edu.cn/agriGO/) was used for gene ontology enrichment. Target genes and functional annotation of differentially expressed genes are given in Dataset Item 3 (Table).

Differential expression analysis was performed as follows. The frequency of miRNAs in control and treatment libraries was normalized to transcripts per million (TPM) by the following formula: normalized expression $=$ (actual miRNA count/total count of clean reads) $\times 1,000,000$ (Dataset Item 4 (Table)). Fold change of miRNA expression between treatment and control libraries was determined and miRNAs with higher than 1.5-fold difference in expression levels were considered to be differentially expressed under drought stress. If the normalized expression of miRNA in both the control and the treatment libraries showed a value of less than one, it is removed due to the very low level of expression. Fold change with positive values indicates upregulation and negative values represent downregulation of miRNAs in the drought treated library.

\section{Dataset Description}

The dataset associated with this Dataset Paper consists of 4 items which are described as follows.

Dataset Item 1 (Table). Details of novel miRNAs identified from control and treated small RNA libraries using miRDeep-P tool.

\section{Column 1: Star Score}

Column 2: Minimum Folding Energy Score

Column 3: Frequency Score 


\section{Column 34: Star Read \\ Column 35: Star Sequence \\ Column 36: Star Structure}

Dataset Item 2 (Table). Targets of all the cardamom miRNAs determined in this study predicted using the psRNATarget software with default parameters. The column Expectation presents the expectation to score the complementarity between miRNA and their target transcript; the column UPE, the maximum energy to unpair the target site; the column miRNA Start, the sequence number of miRNA from which complementarity between miRNA and target transcript starts; the column miRNA End, the sequence number of miRNA in which complementarity between miRNA and target transcript ends; the column Target Start, the sequence number of target transcript from which complementarity between miRNA and target transcript starts; the column Target End, the sequence number of target transcript in which complementarity between miRNA and target transcript ends; the column Inhibition, the mechanism of gene silencing; and the column Multiplicity, the multiplicity of target site.

Column 1: miRNA Sequence
Column 2: Target mRNA ID
Column 3: Expectation
Column 4: UPE
Column 5: miRNA Start
Column 6: miRNA End
Column 7: Target Start
Column 8: Target End
Column 9: miRNA Aligned Fragment
Column 10: Target Aligned Fragment
Column 11: Inhibition
Column 12: Target Description
Column 13: Multiplicity

Dataset Item 3 (Table). Functional annotation of target genes of differentially expressed miRNA families under drought stress. The column miRBase ID presents the miRBase ID of miRNA family and the column Targets presents the target mRNAs and their functional annotation.

Column 1: miRBase ID

Column 2: Targets

Dataset Item 4 (Table). The frequency of miRNAs in control and treatment libraries was normalized to transcripts per million (TPM) and fold change of miRNA expression between treatment and control library was determined. The column Fold Change presents the fold change of miRNA expression between treated library and control library.

Column 1: Name of miRNA

Column 2: Count of miRNA in Control Library
Column 3: Count of miRNA in Treated Library

Column 4: Normalized Reads in Control Library

Column 5: Normalized Reads in Treated Library

Column 6: Fold Change

\section{Concluding Remarks}

Small cardamom is affected by many pests, diseases, and abiotic stress factors that have been identified as major production constraints. If these negative factors can be cut down, cardamom production can still go high. This dataset helps in the identification and characterization of drought responsive miRNAs in cardamom, which would eventually be useful in the development of varieties with a lower requirement of water. This dataset allows researchers to develop microsatellite markers from noncoding regions of cardamom genome.

\section{Dataset Availability}

The dataset associated with this Dataset Paper is dedicated to the public domain using the CC0 waiver and is available at https:/doi.org/10.1155/2017/9507485/dataset. In addition, the sequencing data were deposited in the NCBI Sequence Read Archive (SRA) (https://www.ncbi.nlm.nih.gov/sra/) with accession numbers SRX2273832 and SRX2273833.

\section{Conflicts of Interest}

The authors declare that they have no conflicts of interest.

\section{Authors' Contributions}

N. Anjali and F. Nadiya isolated small RNAs. N. Anjali and Jinu Thomas conducted sequence analysis. N. Anjali performed target identification for the miRNAs and interpretation of the results and prepared the first version of the manuscript. K. K. Sabu designed the experiment, collected the required plant specimens, and prepared the final version of the manuscript.

\section{Acknowledgments}

This research was supported by Kerala State Council for Science, Technology and Environment (KSCSTE), Trivandrum, by granting research fellowship to N. Anjali (01031/FSHP/10/CSTE). The authors would like to thank the Director of the Jawaharlal Nehru Tropical Botanic Garden and Research Institute (JNTBGRI) for providing the necessary facilities to carry out this research work. The authors would also like to acknowledge the permission granted by Kerala Forest Department for collecting the plant samples. Thanks are due to J. S. Vishnu and S. Shefeek for the assistance rendered for the study. The authors are grateful to the staff at the Centre for Cellular and Molecular Platforms (CCAMP, Bangalore, India) for performing high throughput sequencing. 


\section{References}

[1] S. Bagewadi, T. Bobić, M. Hofmann-Apitius, J. Fluck, and R. Klinger, "Detecting miRNA Mentions and Relations in Biomedical Literature," F1000Research.

[2] F. Sun, G. Guo, J. Du et al., "Whole-genome discovery of miRNAs and their targets in wheat (Triticum aestivum L.)," BMC Plant Biology, vol. 14, no. 1, article no. 142, 2014.

[3] S. Bej and J. Basak, "MicroRNAs: The Potential Biomarkers in Plant Stress Response," American Journal of Plant Sciences, vol. 05, no. 05, pp. 748-759, 2014.

[4] G. Akdogan, E. D. Tufekci, S. Uranbey, and T. Unver, "miRNAbased drought regulation in wheat," in Functional Integrative Genomics, pp. 10-1007, 2015.

[5] J. Ferdous, S. S. Hussain, and B.-J. Shi, "Role of microRNAs in plant drought tolerance," Plant Biotechnology Journal, vol. 13, no. 3, pp. 293-305, 2015.

[6] F. Nadiya, N. Anjali, A. Gangaprasad, and K. K. Sabu, "Highquality RNA extraction from small cardamom tissues rich in polysaccharides and polyphenols," Analytical Biochemistry, vol. 485, Article ID 12091, pp. 25-27, 2015.

[7] M. Martin, "Cutadapt removes adapter sequences from highthroughput sequencing reads," EMBnet Journal, vol. 17, no. 1, pp. $10-12,2011$.

[8] B. Langmead, C. Trapnell, M. Pop, and S. L. Salzberg, "Ultrafast and memory-efficient alignment of short DNA sequences to the human genome," Genome Biology, vol. 10, no. 3, article R25, 2009.

[9] X. Yang and L. Li, "miRDeep-P: A computational tool for analyzing the microRNA transcriptome in plants," Bioinformatics, vol. 27, no. 18, Article ID btr430, pp. 2614-2615, 2011.

[10] X. Dai and P. X. Zhao, "PsRNATarget: a plant small RNA target analysis server," Nucleic Acids Research, vol. 39, no. 2, pp. W155W159, 2011. 

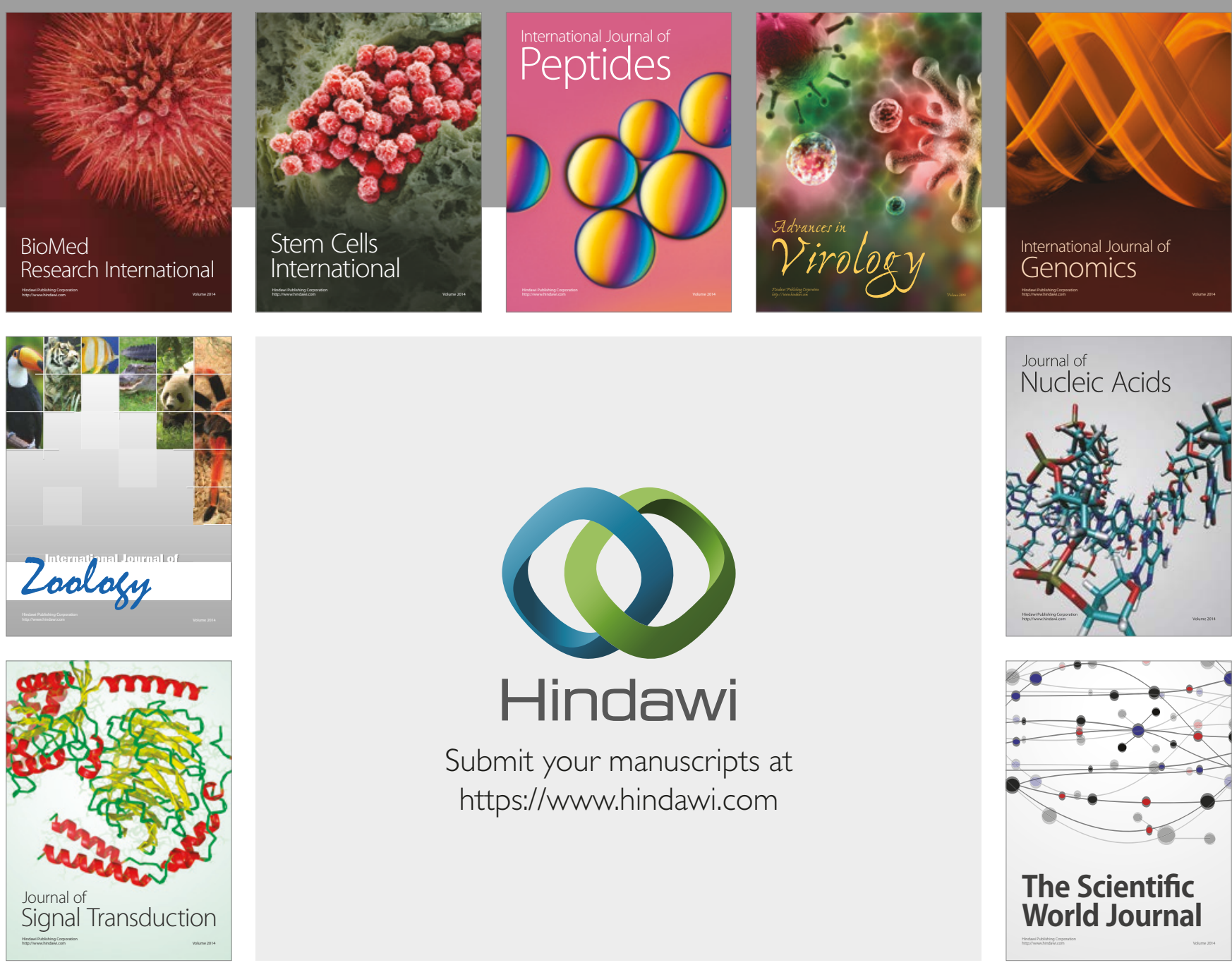

Submit your manuscripts at

https://www.hindawi.com
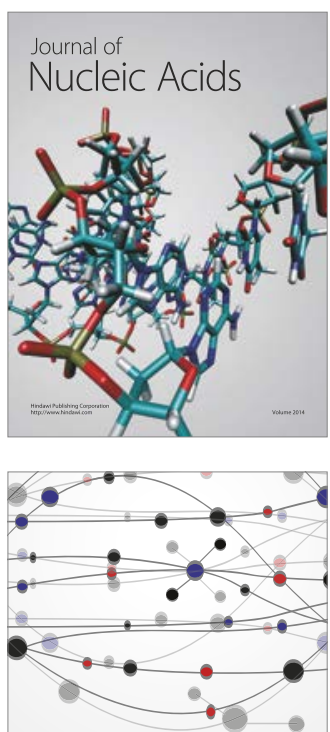

The Scientific World Journal

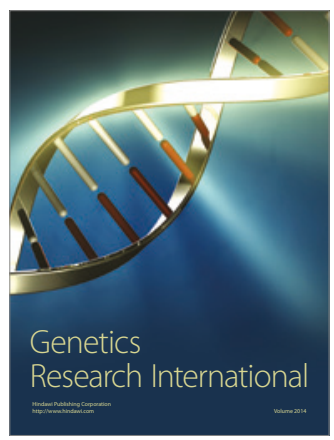

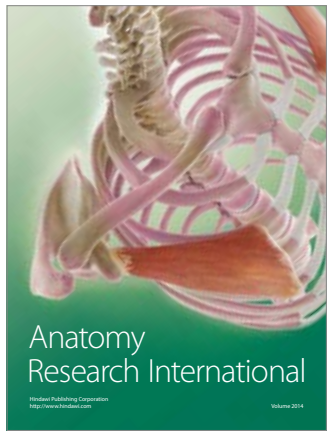

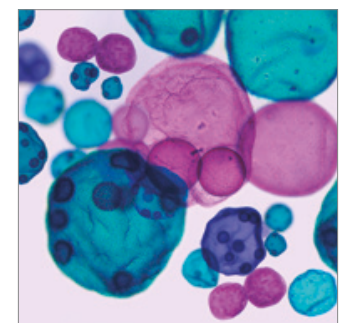

International Journal of Microbiology
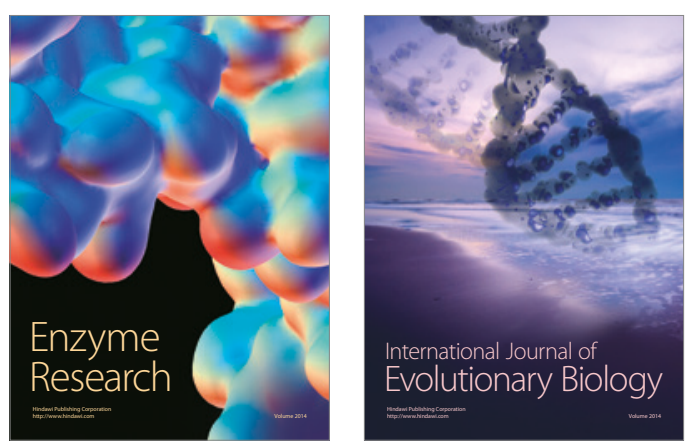
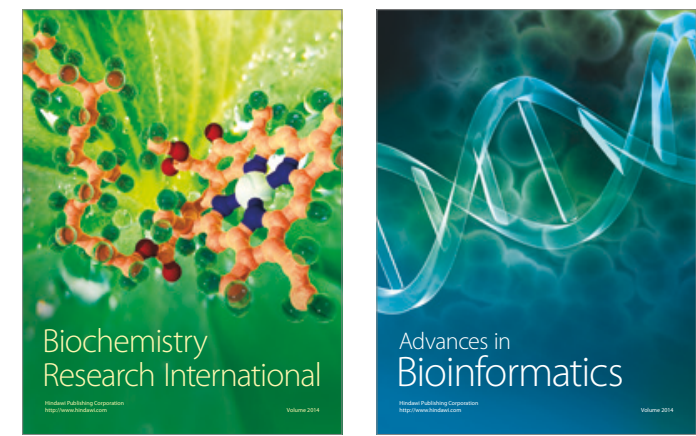

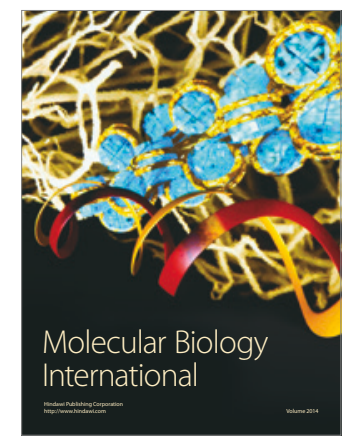

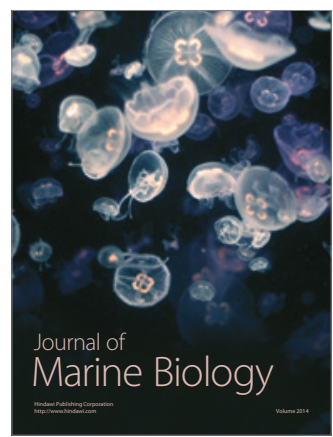

